\title{
Session II: Identifying and Defining Hazards and Potential Consequences I: Concepts for Problem Formulation and Non-Target Risk Assessment
}

\section{Franz BIGLER*}

Agroscope Reckenholz-Taeknikon ART, Swiss Agricultural Research, Reckenholzstrasse 191, 8046 Zurich, Switzerland

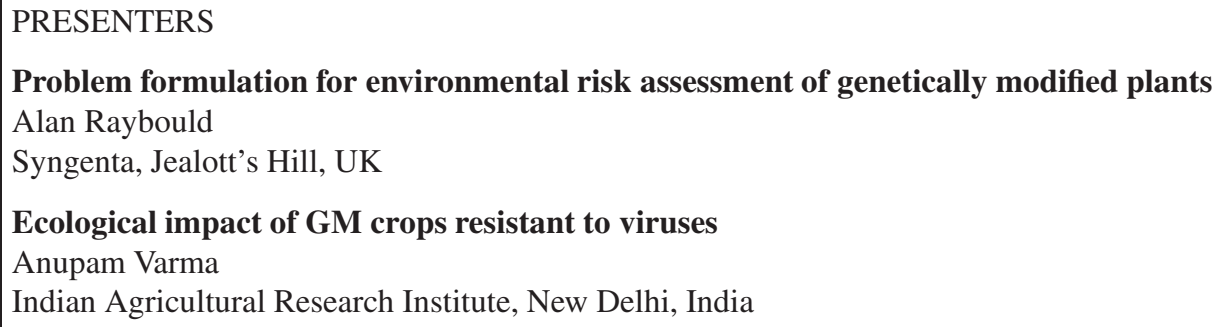

The scientific organizers of the symposium put much emphasis on the identification and definition of hazard and the potential consequences thereof and three full sessions with a total of 13 presentations encompassing a wide range of related themes were planned for this topic. Unfortunately, one talk had to be cancelled because of illness of the speaker (BM Khadi, India). Some presentations covered conceptual approaches for environmental risk assessment (ERA) of GM plants (problem formulation in the risk assessment framework, familiarity approach, tiered and methodological frameworks, non-target risk assessment) and the use of models in assessing invasiveness and weediness of GM plants. Other presentations highlighted the lessons learned for future ERA from case studies and commercialized GM crops, and from monitoring of unintended releases to the environment. When the moderators of the three sessions came together after the presentations to align their summaries, there was an obvious need to restructure the 12 presentations in a way that allowed for a consistent summarizing discussion. The following new organization of the 12 talks was chosen:

(1) Concepts for problem formulation and non-target risk assessment

(2) Modeling as a tool for predicting invasiveness of GM plants

(3) Case-studies of ERA of large-scale release

(4) Lessons learned for ERA from a commercialized GM plant

(5) Monitoring of unintended release of Bt maize in Mexico.

The new thematic structure facilitates a more in-depth discussion of the presentations related to a specific topic, and the conclusions to be drawn are thus more consistent. Each moderator agreed to take responsibility for summarizing one or more themes and to prepare the respective report.

* Corresponding author: franz.bigler@art.admin.ch 


\section{INTRODUCTION}

While conceptual frameworks for addressing risks associated with environmental stressors, such as chemicals (e.g. pesticides) and other environmental stressors (e.g. invasive species) are near-universal, there is not yet unanimous agreement on a conceptual approach for genetically modified (GM) plants. Biodiversity and nontarget impacts play a major role in the environmental risk assessment of pest resistant and herbicide tolerant GM plants, and it is thus crucial for industry, GM plant developing public institutes and regulators to have consensus on the principles of addressing such potential risks. The symposium offered a remarkable platform for presenting different aspects of such principles and discussing views of conceptual approaches.

The bases for this report and synthesis are mainly the written manuscripts in the proceedings of the symposium, however, the general considerations given in this overview reflect the personal view of the author.

\section{PROBLEM FORMULATION, THE INITIAL POINT IN A TIERED FRAMEWORK FOR RISK ASSESSMENT}

All presentations and the subsequent discussions made clear that the general concepts for risk assessment of GM plants entails a tiered and iterative approach, whatever terminology is used and numbers of steps adopted. At the end of the day, there was a general consensus among participants that problem formulation should be the first step in the risk assessment process, where all existing information on the GM plant, the stressor(s) (e.g. toxin) and the possible receiving environments are gathered and surveyed to identify potential effects on the environment.

Critical to any problem formulation is the development of a conceptual model, a detailed analysis plan including assessment endpoints, and hazard and exposure identification. A structured conceptual model in which the components of the system (plant-stressorenvironment-hazard-exposure, e.g. for non-target organisms) are put in a detailed flow path will allow the identification and formulation of relevant hypotheses. The immediate action in problem formulation is normally a review summarizing existing knowledge of the system, including published and unpublished data. The resulting information focuses to the relevant problems and hypothetical risks thereof. The conceptual model specifies the assessment endpoints, i.e. those components in the environment that are important to protect and that are potentially at risk. Given that general protection goals (e.g. health of the environment) cannot be measured as such, assessment endpoints should be as specific as possible and indicative of the protection goals and the system they represent. For example, in a risk assessment of plants expressing insecticidal proteins, a measurable protection goal could be biological control function in the crop. In this case, a logical assessment endpoint would be represented by the population sizes of (a few) selected nontarget organism species that are important for biological control. Most assessment endpoints are not directly measured, instead, other characteristics, so called measurement endpoints, are determined.

The analysis plan specifies the details of testing the formulated hypotheses, by adopting appropriate measurement endpoints that relate quantifiably to the assessment endpoints. The plan outlines the measures to be used, the characterization of effects, exposure and expected risks. In the case of assessing biological control function, appropriate test organisms (surrogate species) are selected, and tests are designed including the detailed information on measurement endpoints (such as mortality, fecundity, etc.), the analyses of assessed data, the statistical power to be achieved, and all other tools that will later be used in the hazard assessment.

Another important part of problem formulation is hazard and exposure identification. Conceptually, hazard is the intrinsic toxicity of a stressor. Hazard identification is about potential hazards to an organism or specific life stages, based on existing information such as spectrum and scope of activity, mode of action and sensitivity of life stages. Exposure identification is about plausible routes of exposure of a biological entity to the stressor. In the case of biological control function, possible exposure is identified based on known expression of the toxin in different plant parts at varying times and levels, feeding habits (prey/host spectrum, plant feeding) of relevant natural enemies and their life stages.

Why is the distinction of the different components in problem formulation so important? The identification of potential risks based on all available information sources is a complex process that needs to be structured and set in a logical sequence, in order to make the risk assessment transparent and comprehensive, and to allow regulatory authorities to make decisions. In the context of regulation of GM plants, it is important to keep in mind that the purpose of risk assessment is to contribute to decisionmaking, and not to broaden knowledge in general ("nice to know and need to know"). Without the distinction of the different steps in the problem formulation, risk assessment would be much less focused, and may not identify the most important questions.

\section{THE CONCEPT OF FAMILIARITY IN THE CONTEXT OF RISK ASSESSMENT}

Another important source of information for decisionmakers is implied by the concept of familiarity of GM 
plants. Pre-existing knowledge, experimental results, expert opinions and experience gained over time with GM crops relative to traditional crops are needed for risk assessment. Familiarity encompasses the characterization of the crop, the trait, the likely receiving environments and the potential interactions among them. Given that familiarity increases with time and experience, it helps to reduce uncertainty and to direct more focused information collection. Familiarity is based on a comparative assessment approach that considers the GM crop in the context of appropriate non-GM controls and commercial reference varieties. This provides information about the variation for a specific character, and whether the GM plant lies within the normal variation. Information gathered enables the risk assessor to determine with greater certainty and precision those characteristics of the GM plant that are different from the conventional crop and may be of regulatory concern. Methods include comparative compositional analysis and agronomic/phenotypic characterizations of the GM crop relative to the non-GM crop and commercial reference varieties. The concept of familiarity allows regulators to draw on past experience and the risk assessment may be expedited.

\section{NON-TARGET RISK ASSESSMENT FOR REGULATORY PURPOSES}

Conceptual frameworks are critical in risk assessment, as they can provide common understanding for regulators, registrants and scientists, and help to direct the limited resources to the relevant questions. A tiered framework provides a suitable approach for addressing relevant questions, it organizes and guides the studies that are required during the regulatory process, and it potentially eliminates unneeded studies. A tiered risk assessment is recognized to be the most appropriate and rigorous approach to assess non-target and biodiversity effects from both scientific and regulatory standpoints. Both hazard and exposure can be evaluated within different tiers that progress from worst-case hazard and exposure to more realistic scenarios. Lower tier tests are used to identify potential hazard, and they are conducted in the laboratory to provide high levels of replication in relatively simple study designs and conducted under the rules of Good Laboratory Practice (GLP). Higher tier tests are triggered when laboratory studies indicate potential hazard at relevant environmental doses taking into account safety factors. In cases where potential hazard is detected in lower tier tests, the iterative approach of a tiered framework provides the flexibility to conduct further lower tier tests or to progress to subsequent higher tier testing with more realistic and complex designs, depending on the nature of effects, and in cases where laboratory tests would not provide sufficient data for making decisions. A tiered approach is intended to provide enough flexibility to address changing testing and assessment needs on a case by case basis. As a principle, rather extensive testing is conducted at lower tiers, and further testing is restricted and focused on specific issues of residual uncertainty arising out of the previous tier assessments. Therefore, regulatory decisions are achieved without the need for every GM plant to proceed through each tier. A tiered approach is designed to optimize the use of resources, focus and direct risk assessment to relevant questions, and to provide sufficient high quality data to regulators to allow them decision-making with high certainty.

Most tiered approaches that have been elaborated for non-target risk assessment of GM plants follow the same underlying principles, although they may differ in the definitions of specific tiers. A different approach has been proposed by a group of authors. Although the structure of their approach is also tiered, the tiers are substantially different from those currently used by risk assessors. The process is initiated by the selection of risk endpoints by specifying the receiving crop environment and listing relevant ecological functional groups so that the biological diversity of the ecosystem is simplified into smaller numbers of functional categories. For each of these functional groups, a characteristic risk endpoint is identified. The next step is to list relevant species and ecosystem processes within each selected functional group. A series of qualitative ecological characteristics can be used to rank the species in relation to the likelihood of the risk endpoint associated with the functional group. The selection of the risk endpoints is a process that relies on expert judgement about the crop, the kind of GM trait and the receiving environment. However, it does not rely on specific information about the transgene or transgene product.

Subsequent to the selection of risk endpoints, the methodology develops risk hypotheses to facilitate risk characterization. Risk hypotheses are defined as being complex interconnected nets of multiple chains because there are multiple pathways by which a GM crop could generate an adverse effect. Coupled with knowledge about the receiving environment and the selected species or processes, the authors propose to represent in a diagram all of the pathways by which the GM crop may result in an adverse effect for a given risk endpoint and species or ecosystem process. Subsequently, risk hypotheses are analysed to identify the strength of the links, so that most efforts can be targeted to the high priority links for risk assessment research.

The proposed methodology provides four tiers to assess risks to biological diversity in a particular receiving environment. Tier 1 is the determination of relevant functional groups, corresponding to the first part of the identification of risk endpoints. Groups that are excluded are 
deemed irrelevant because there is no risk associated with them. Tier 2 is the selection of species and processes considered most associated with a risk endpoint. This tier reduces the potential candidates for further risk assessment, because the unselected species and processes are judged to be at lower risk than the selected ones. Tier 3 is a qualitative assessment of the risk hypotheses considering both direct and indirect effects. In this tier it may be possible to determine that the risk to several of the selected species and processes in unlikely and small. Hence, this tier removes specific risk hypotheses, but the ecological entity may still be affected via other risk hypotheses. Tier 4 is the quantitative assessment and could include toxicity tests and other laboratory tests, semi/field and field trials. Without giving any specifications, the authors note that several alternative evaluation processes may be possible that could efficiently evaluate the risk hypotheses. The most appropriate sequence of tests may be designed for the settings available in each country, depending on the specific situation.

\section{CONCLUSIONS}

The tiered risk assessment approach for GM plants adopted by regulatory authorities makes use of the nearuniversal principles which are used for risk assessments in other disciplines, e.g. pesticides, biological control agents and invasive organisms. Hence, authorities can refer to the long standing experience in environmental risk assessment and management. Registrants and risk assessors should utilise all available information that is accessible, follow a transparent structure during the risk assessment and come to decisions within a reasonable time by applying adequate resources. The principles of the tiered approach as it is presently applied by registrants and regulatory authorities, and advocated by most scientists performing risk assessment research, is best qualified to fulfil the needs of regulatory authorities and to ensure the safe use of GM crops in the future. 